\title{
Monocyte to high-density lipoprotein cholesterol ratio is predictive of in-hospital and five-year mortality in ST-segment elevation myocardial infarction
}

\author{
Sadık Kadri Açıkgöz ${ }^{1}$, Eser Açıkgöz ${ }^{2}$, Barış Şensoy ${ }^{1}$, Salih Topal ${ }^{3}$, Sinan Aydoğdu ${ }^{1}$ \\ ${ }^{1}$ Department of Cardiology, Turkiye Yuksek Ihtisas Education and Research Hospital, Ankara, Turkey \\ ${ }^{2}$ Department of Cardiology, Ankara Abdurrahman Yurtaslan Oncology Education \\ and Research Hospital, Ankara, Turkey \\ ${ }^{3}$ Department of Cardiology, Gazi University School of Medicine, Ankara, Turkey
}

\begin{abstract}
Background: We assessed the value of monocyte to high-density lipoprotein cholesterol ratio (MHR) in predicting in-hospital and 5-year mortality and major adverse cardiovascular events (MACE) in ST-segment elevation myocardial infarction (STEMI) patients.

Methods: A group of 1,598 patients were enrolled and divided into tertiles according to MHR values. The effects of different variables on clinical outcomes were assessed by Cox regression analysis. Results: MHR was found as an independent predictor of in-hospital mortality (HR $=3.745$, 95\% CI 1.308-5.950), in-hospital MACE (HR 1.501, 95\% CI 1.015-1.993, p = 0.022) and 5-year mortality $(H R=2.048,95 \%$ CI 1.225-4.091, $p=0.014)$ and 5-year MACE (HR $1.285,95 \%$ CI 1.064-1.552, $p=0.009)$.
\end{abstract}

Conclusions: MHR is an independent predictor of in-hospital and long term mortality and MACE in STEMI. (Cardiol J 2016; 23, 5: 505-512)

Key words: monocyte to high-density lipoprotein ratio, ST-segment elevation myocardial infarction, monocyte, high density lipoprotein cholesterol

\section{Introduction}

It is well known that inflammation and oxidative stress play a crucial role in the pathogenesis of all phases of atherosclerosis [1, 2]. Among the subtypes of leucocytes, monocytes are especially important in the pathogenesis of atherosclerosis due to their secretion capability of pro-inflammatory and pro-oxidant cytokines [3]. In contrast, high density lipoprotein cholesterol (HDL-C) was shown to have anti-inflammatory, anti-oxidant, and anti-thrombotic effects $[4,5]$. Association of high monocyte count and low HDL-C with inflammation and oxidative stress led physicians to testing a new marker called monocyte to high-density lipoprotein cholesterol ratio (MHR) in cardiovascular conditions. First, Kanbay et al. [6] found that increased MHR is associated with major cardiovascular events in chronic kidney disease. Subsequent studies revealed the association of MHR with atrial fibrillation recurrence after cryoballoon-based catheter ablation, coronary slow flow, coronary artery ectasia and stent thrombosis after ST-segment elevation myocardial infarction (STEMI) [7-10].

Address for correspondence: Sadık Kadri Açıkgöz, MD, TurkiyeYuksek Ihtisas Hastanesi, 06100, Sihhiye, Ankara, Turkey, tel: +90 312 3061134, fax: +90 3123124120 , e-mail: drskad@gmail.com

Received: 12.01.2016 Accepted: 07.05.2016 
Recently, Karataş et al. [11] demonstrated that MHR values were independently associated with in-hospital mortality in patients with STEMI. However, value of the MHR in prediction of long-term prognosis of STEMI patients has not been studied so far. Therefore, the aim of the present study was to investigate the relationship of MHR with in-hospital and 5-year major adverse cardiac events (MACE) and mortality of STEMI patients managed with primary percutaneous coronary intervention (PCI).

\section{Methods}

\section{Study population}

One thousand, five hundred and ninety-eight consecutive patients with STEMI, who were admitted to the Emergency Department and underwent urgent cardiac catheterization between December 2009 and December 2013, were enrolled into the study. Patients with acute infection, hematological diseases, malignancy, and chronic systemic disease were excluded from the study. The patients were divided into tertiles according to their MHR levels. STEMI was defined as ischemia symptoms and $\geq 1 \mathrm{~mm}$ ST-segment elevation of in at least 2 contiguous electrocardiogram (ECG) leads except V2-V3 which required $1.5 \mathrm{~mm}$ for female patients, $2 \mathrm{~mm}$ for male patients $>40$ years old, and $2.5 \mathrm{~mm}$ for male patients $<40$ years old or new onset left bundle-branch block. The study protocol was approved by the Institutional Ethics Committee.

\section{Analysis of patient data}

The demographic information, medical history, and cardiovascular risk factors of the patients were obtained from their medical records. On admission, blood values were obtained from all patients. A 12-lead ECG was recorded in each patient just after hospital admission, and the myocardial infarction type was also obtained from the ECGs. From $24 \mathrm{~h}$ to $72 \mathrm{~h}$ after revascularization, a transthoracic echocardiography was performed by using a system V (Vingmed, GE, Horten, Norway) with a $2.5-\mathrm{MHz}$ phased-array transducer, and the left ventricular ejection fraction (LVEF) was calculated using a modified Simpson's method.

\section{Coronary angiography and PCI}

All patients were given a chewable $300 \mathrm{mg}$ aspirin and $600 \mathrm{mg}$ loading dose of clopidogrel before coronary angiography. After the procedure, all patients were prescribed $100 \mathrm{mg}$ aspirin and $75 \mathrm{mg}$ clopidogrel daily. Angiographic data of the patients were assessed from catheter laboratory records. All procedures were performed via femoral route. Angiographic evaluation was made by visual assessment. Primary angioplasty (including balloon angioplasty and/or stent implantation) was performed just for infarct-related artery according to lesion type. For each procedure, interventional success at the acute phase was defined as reducing to $<30 \%$ of obstruction and stenosis of the infarct-related artery with Thrombolysis in Myocardial Infarction (TIMI) 3 flow just after primary angioplasty. The use of tirofiban was left to the discretion of the operator.

\section{Laboratory analysis}

Basic hematologic parameters, such as monocyte count were determined by a Coulter LH Series hematology analyzer (Beckman Coulter, Inc, Hialeah, Florida). Serum total cholesterol, triglyceride, and HDL-L concentrations were analyzed by a BMHitachi-747 auto analyzer (Boehringer Mannheim $\mathrm{GmbH}$, Mannheim, Germany). Serum low-density lipoprotein cholesterol (LDL-L) values were estimated by the formula of Friedewald or directly measured if triglyceride $>400 \mathrm{mg} / \mathrm{dL}$.

\section{Definitions}

Patients were assessed according to Killip classification [14]. Non-diabetic patients were defined as patients without documented diabetes using neither oral hypoglycemic agents nor insulin treatment at admission. Hypertension was defined as systolic blood pressure $\geq 140 \mathrm{~mm} \mathrm{Hg}$ or diastolic blood pressure $\geq 90 \mathrm{~mm} \mathrm{Hg}$ or current antihypertensive medicine use; a family history of coronary artery disease was defined as documented case of coronary artery disease in a parent or sibling before 60 years of age; smoking was defined as current tobacco use; in-hospital mortality was defined as mortality due to any cause during hospital stay, and long-term mortality was defined as mortality due to any cause during 60 month follow up; in-hospital MACE was defined as reinfarction, stroke, target vessel revascularization, ventricular arrhythmia, cardiopulmonary resuscitation and death during index hospitalization; long term MACE was defined as death, stroke, reinfarction and target vessel revascularization during 60 -month follow-up.

\section{Statistical analysis}

Analyses were performed using SPSS Statistics version18.0 for Windows (SPSS Inc, Chicago, IL). The Kolmogorov-Smirnov method was used to test the distribution pattern. Data were presented as mean and standard deviation, median and in- 
terquartile range, or proportions. The one way ANOVA was used to compare data with normal distribution and Kruskal-Wallis test was applied to compare the data without normal distribution. Categorical variables were compared with the $\chi^{2}$ test. The effects of different variables on clinical outcomes were assessed by Cox regression analysis. The variables for which the unadjusted $p$ value was $<0.10$ in univariate Cox regression analysis were identified as potential risk markers and included in the multivariable Cox regression model. Monocyte to HDL ratio was used as a continuous variable rather than tertiles in Cox regression models. The survival curves during hospitalization and follow-up for MHR were analyzed using the Kaplan-Meier method, and statistical assessment was performed using the log-rank test. A p value $<0.05$ was considered statistically significant for all analyses.

\section{Results}

Baseline characteristics of the study population-are summarized in Table 1 . Mean age of the participants was $56.1 \pm 11.8$ years and mean follow-up duration was $23.4 \pm 15.8$ months. Twenty-three (1.4\%) patients died during hospital stay and $132(8.7 \%)$ patients died during the follow-up. In multivariate $\mathrm{Cox}$ regression analysis, age $(\mathrm{HR}=$ $=1.144,95 \%$ CI $1.067-1.227, \mathrm{p}=0.001), \mathrm{HDL}$ $(\mathrm{HR}=0.894,95 \%$ CI 0.819-0.977, $\mathrm{p}=0.013)$, white blood cell $(\mathrm{WBC})$ count $(\mathrm{HR}=1.258,95 \%$ CI 1.010-1.459), and MHR (HR $=3.745,95 \%$ CI 1.308-5.950) were found as independent predictors of in-hospital mortality (Table 2). Monocyte to HDL ratio was also found as an independent predictor of long-term mortality ( $\mathrm{HR}=2.048,95 \%$ CI 1.225-4.091, $\mathrm{p}=0.014)$ along with diabetes mellitus $(\mathrm{HR}=2.279,95 \%$ CI 1.197-4.340, $\mathrm{p}=$ $=0.012), \mathrm{LVEF}(\mathrm{HR}=0.958,95 \%$ CI $0.938-0.978$, $\mathrm{p}<0.001)$, and hemoglobin $(\mathrm{HR}=0.795,95 \%$ CI $0.679-0.930, \mathrm{p}=0.004$ ) (Table 3 ), while WBC count $(\mathrm{HR}=1.073,95 \% \mathrm{CI} 1.000-1.152, \mathrm{p}=0.049)$ and MHR (HR 1.501, 95\% CI 1.015-1.993, $\mathrm{p}=0.022$ ) were found as independent predictors of in-hospital MACE (Table 4). In addition, LVEF (HR 0.980, 95\% CI 0.967-0.993, $\mathrm{p}=0.003$ ), hemoglobin (HR 0.909, 95\% CI 0.829-0.996, $\mathrm{p}=0.041$ ), and MHR (HR $1.285,95 \%$ CI $1.064-1.552, \mathrm{p}=0.009$ ) were found as independent predictors of long-term MACE (Table 5). Kaplan-Meier curves among tertiles for both in-hospital and 5-year MACE and mortality, which represent worse outcomes as MHR increases, were shown in Figure 1.

\section{Discussion}

The main finding of the present study was that MHR level is independently associated with both in-hospital and 5-year MACE, and mortality of STEMI patients. As far as we know, this is the first study to report relationship of MHR with long-term prognosis in STEMI. In addition, findings of this study confirm a previous study with smaller patient count [11] revealing the association of in-hospital mortality and MACE with MHR in STEMI.

Monocytes are essential immune system cells with unique roles during inflammatory response and contribute the pathophysiology of all stages of atherosclerosis. In the presence of endothelial dysfunction, monocytes attach to endothelium and migrate to sub-endothelial space where they mature into macrophages which then differentiate into the foam cells by taking up oxidized LDL via scavenger receptors SR-A and CD-36 [12]. Next, foam cells secrete pro-inflammatory cytokines, matrix metalloproteinases, growth factors and tissue factor [13]. As a result, smooth muscle proliferation occurs and results in plaque growth. Furthermore, matrix metalloproteinases inflict damage to elastic lamina and predispose to plaque rupture. If the plaque rupture occurs, tissue factor acts as a co-factor for factor VII and causes thrombin production resulting in thrombus formation and acute coronary syndromes [14]. Impact of the monocytes on atherosclerosis seems to be correlated with circulating monocyte count which was found to be predictive of new plaque development $[15,16]$.

As the so-called good cholesterol, HDL-C protects endothelial cells from inflammation and oxidative stress by several mechanisms. First, HDL-C prevents monocyte recruitment into the artery wall by inhibiting the expression of endothelial adhesion molecules [17]. In addition, HDL-C has a role in control of the monocyte activation and proliferation of monocyte progenitor cells [18-20]. Furthermore, HDL-C inhibits oxidation of LDL-C and promotes efflux of oxidized LDL-C from foam cells $[5,18]$.

Association of HDL-C and inflammation is not just about the anti-inflammatory action of HDL-C. During inflammation, there is a reduction in levels of HDL-C and some plasma proteins are involved in HDL-mediated reverse cholesterol transport and inhibiting plasma lipid oxidation, such as apolipoprotein A-I and paraoxonase [21]. In addition, HDL-C loses cholesterol ester but gains free cholesterol, triglyceride, and free fatty acids [22]. The levels 
Table 1. Clinical and laboratory characteristics of monocyte to high-density lipoprotein cholesterol ratio (MHR) tertiles.

\begin{tabular}{|c|c|c|c|c|}
\hline \multirow[t]{2}{*}{ Variables } & \multicolumn{3}{|c|}{ MHR } & \multirow[t]{2}{*}{$\mathbf{P}$} \\
\hline & Tertile $1(n=533)$ & Tertile $2(n=533)$ & Tertile $3(n=532)$ & \\
\hline Age [years] & $58.2 \pm 12.2$ & $55.9 \pm 11.4$ & $54.4 \pm 11.5$ & $<0.001$ \\
\hline Gender (male) & $423(79.4 \%)$ & $455(85.4 \%)$ & $463(87.0 \%)$ & 0.002 \\
\hline Diabetes mellitus & $130(24.5 \%)$ & $110(20.9 \%)$ & $138(26.1 \%)$ & 0.125 \\
\hline Hypertension & $210(41.5 \%)$ & $196(38.4 \%)$ & $200(39.6 \%)$ & 0.587 \\
\hline Family history of CAD & $103(21.1 \%)$ & $94(18.8 \%)$ & $93(28.9 \%)$ & 0.573 \\
\hline Smoking & $267(54.9 \%)$ & $325(65.3 \%)$ & $332(67.5 \%)$ & $<0.001$ \\
\hline Ejection fraction [\%] & $48.9 \pm 10.7$ & $48.1 \pm 11.4$ & $46.9 \pm 11.7$ & 0.121 \\
\hline Glucose [mg/dL] & $156.7 \pm 68.8$ & $148.5 \pm 68.9$ & $151.5 \pm 67.5$ & 0.143 \\
\hline Creatinine [mg/dL] & $0.95 \pm 0.45$ & $0.97 \pm 0.31$ & $0.98 \pm 0.34$ & 0.348 \\
\hline Cholesterol [mg/dL] & $189.7 \pm 42.3$ & $192.1 \pm 43.5$ & $184.4 \pm 41.7$ & 0.011 \\
\hline LDL [mg/dL] & $118.9 \pm 35.5$ & $118.8 \pm 33.9$ & $115.6 \pm 35.8$ & 0.257 \\
\hline $\mathrm{HDL}[\mathrm{mg} / \mathrm{dL}]$ & $43.6 \pm 10.2$ & $40.7 \pm 8.1$ & $38.1 \pm 8.2$ & $<0.001$ \\
\hline Triglycerides [mg/dL] & $141.2 \pm 104.4$ & $154.4 \pm 109.1$ & $151.4 \pm 82.1$ & 0.093 \\
\hline Hemoglobin [g/L] & $13.4 \pm 1.7$ & $13.8 \pm 1.6$ & $13.9 \pm 1.6$ & $<0.001$ \\
\hline WBC $\left[\times 10^{3} / \mathrm{mL}\right]$ & $11.7 \pm 3.4$ & $12.0 \pm 3.2$ & $13.9 \pm 3.9$ & $<0.001$ \\
\hline Monocyte $\left[\times 10^{3} / \mathrm{mL}\right]$ & $0.374 \pm 0.159$ & $0.798 \pm 0.195$ & $1.018 \pm 0.471$ & $<0.001$ \\
\hline Platelets $\left[\times 10^{3} / \mathrm{mL}\right]$ & $256.0 \pm 73.8$ & $250.9 \pm 62.5$ & $272.5 \pm 74.6$ & $<0.001$ \\
\hline MHR & $8.63 \pm 3.10$ & $19.8 \pm 3.8$ & $30.1 \pm 10.5$ & $<0.001$ \\
\hline \multicolumn{5}{|c|}{ Postprocedural TIMI grade: } \\
\hline 1 & $45(8.4 \%)$ & $39(73 \%)$ & $50(9.4 \%)$ & 0.735 \\
\hline 2 & $24(4.5 \%)$ & $27(5.1 \%)$ & $23(4.3 \%)$ & \\
\hline 3 & $464(87.1 \%)$ & $467(87.6 \%)$ & $459(86.3 \%)$ & \\
\hline Peak CK-MB [U/L] & $208 \pm 145$ & $215 \pm 171$ & $231 \pm 184$ & 0.073 \\
\hline \multicolumn{5}{|l|}{ Intervention: } \\
\hline Balloon angioplasty & $16(3.0 \%)$ & $16(3.0 \%)$ & $16(3.0 \%)$ & 0.465 \\
\hline BMS & $149(28.0 \%)$ & $147(27.6 \%)$ & $142(26.7 \%)$ & \\
\hline DES & $368(69.0 \%)$ & $370(69.4 \%)$ & $375(70.3 \%)$ & \\
\hline Tirofiban use & $145(27.2 \%)$ & $156(29.3 \%)$ & $158(29.7 \%)$ & 0.598 \\
\hline Pain to balloon time [h] & $3.3 \pm 2.3$ & $3.1 \pm 2.2$ & $3.2 \pm 2.5$ & 0.636 \\
\hline In-hospital MACE & $24(4.5 \%)$ & $44(8.2 \%)$ & $59(11.1 \%)$ & $<0.001$ \\
\hline 5-year MACE & $114(21.3 \%)$ & $135(25.3 \%)$ & $169(31.8 \%)$ & 0.001 \\
\hline In-hospital mortality & $5(0.9 \%)$ & $6(1.1 \%)$ & $17(3.2 \%)$ & 0.008 \\
\hline 5-year mortality & $27(5.1 \%)$ & $43(8.1 \%)$ & $62(11.7 \%)$ & $<0.001$ \\
\hline
\end{tabular}

BMS - bare metal stent; CAD — coronary artery disease; CK-MB — creatine kinase myocardial band; DES — drug eluting stent; HDL — high density lipoprotein; LDL — low density lipoprotein; MACE — major adverse cardiac events; TIMI — Thrombolysis in Myocardial Infarction; WBC - white blood cell

of apolipoprotein $\mathrm{J}$ and serum amyloid-A increase several fold in this "acute-phase HDL" [23]. Acute phase HDL behaves differently from normal HDL and was shown to be less effective in removing cholesterol from macrophages [24]. Thereforeo, HDL-C is a negative acute phase reactant and lower HDL-C levels may reflect more inflammation, as well as less anti-inflammatory activity.
Value of the MHR has been revealed in several cardiovascular conditions so far [6-11]. In addition, our study showed that MHR was found to be an independent predictor of long-term prognosis of STEMI patients, while such an association was not present for monocyte or HDL-C alone. Thus, as a combination of two parameters related with inflammation and oxidative stress, MHR may have potential to be 
Table 2. Significant predictors of in-hospital mortality in univariable and multivariable Cox regression analyses.

\begin{tabular}{|c|c|c|c|c|}
\hline \multirow[t]{2}{*}{ Variables } & \multicolumn{2}{|c|}{ Univariable } & \multicolumn{2}{|c|}{ Multivariable } \\
\hline & HR (95\% CI) & $\mathbf{P}$ & $\mathrm{HR}(95 \% \mathrm{CI})$ & $\mathbf{P}$ \\
\hline Age & $1.129(1.031-1.236)$ & 0.009 & $1.144(1.067-1.227)$ & 0.001 \\
\hline Male gender & $1.483(0.198-11.116)$ & 0.701 & - & - \\
\hline Diabetes mellitus & $1.259(0.254-6.211)$ & 0.778 & - & - \\
\hline Hypertension & $0.568(0.238-1.354)$ & 0.202 & - & - \\
\hline Smoking & $0.209(0.027-1.640)$ & 0.136 & - & - \\
\hline Glucose & $1.002(0.996-1.009)$ & 0.477 & - & - \\
\hline Ejection fraction & $0.969(0.913-1.028)$ & 0.291 & - & - \\
\hline Cholesterol & $1.015(0.992-1.038)$ & 0.203 & - & - \\
\hline HDL & $0.845(0.752-0.949)$ & 0.005 & $0.894(0.819-0.977)$ & 0.013 \\
\hline Triglyceride & $0.991(0.977-1.005)$ & 0.191 & - & - \\
\hline WBC & 1.241 (1.007-1.529) & 0.043 & $1.258(1.010-1.459)$ & 0.028 \\
\hline Monocyte & $1.629(0.956-2.777)$ & 0.072 & $1.815(0.902-2.964)$ & 0.096 \\
\hline Hemoglobin & $0.745(0.448-1.239)$ & 0.257 & - & - \\
\hline Platelet & $1.006(0.999-1.014)$ & 0.093 & $1.005(0.998-1.011)$ & 0.171 \\
\hline Peak CK-MB & $1.003(0.999-1.006)$ & 0.142 & - & - \\
\hline MHR & $3.120(3.058-3.184)$ & 0.003 & $3.745(1.308-5.950)$ & 0.008 \\
\hline
\end{tabular}

$\mathrm{Cl}$ - confidence interval; $\mathrm{CK}-\mathrm{MB}$ - creatine kinase myocardial band; $\mathrm{HDL}$ - how density lipoprotein cholesterol; HR - hazard ratio; $\mathrm{MHR}$ - monocyte to high density lipoprotein cholesterol ratio; WBC - white blood cell

Table 3. Significant predictors of mortality at 5-year follow-up in univariable and multivariable Cox regression analyses.

\begin{tabular}{|c|c|c|c|c|}
\hline \multirow[t]{2}{*}{ Variables } & \multicolumn{2}{|c|}{ Univariable } & \multicolumn{2}{|c|}{ Multivariable } \\
\hline & HR (95\% CI) & $\mathbf{P}$ & HR (95\% CI) & $\mathbf{P}$ \\
\hline Age & $1.018(0.989-1.047)$ & 0.220 & - & - \\
\hline Male gender & $1.426(0.728-2.794)$ & 0.300 & - & - \\
\hline Diabetes Mellitus & $2.012(1.039-3.894)$ & 0.038 & $2.279(1.197-4.340)$ & 0.012 \\
\hline Hypertension & $1.215(0.853-1.731)$ & 0.281 & - & - \\
\hline Smoking & $0.959(0.541-1.702)$ & 0.887 & - & - \\
\hline Glucose & $1.003(1.000-1.006)$ & 0.091 & $1.003(0.999-1.006)$ & 0.116 \\
\hline Ejection fraction & $0.959(0.938-0.980)$ & $<0.001$ & $0.958(0.938-0.978)$ & $<0.001$ \\
\hline Cholesterol & $0.994(0.968-1.002)$ & 0.156 & - & - \\
\hline HDL & $1.023(0.990-1.057)$ & 0.167 & - & - \\
\hline Triglyceride & $1.001(0.998-1.004)$ & 0.600 & - & - \\
\hline WBC & $1.026(0.949-1.109)$ & 0.525 & - & - \\
\hline Monocyte & $1.584(0.945-2.532)$ & 0.164 & - & - \\
\hline Hemoglobin & $0.8500 .705-1.024)$ & 0.087 & $0.795(0.679-0.930)$ & 0.004 \\
\hline Platelet & $1.000(0.996-1.004)$ & 0.920 & - & - \\
\hline Peak CK-MB & $1.000(0.998-1.001)$ & 0.713 & - & - \\
\hline MHR & $2.472(1.547-3.952)$ & $<0.001$ & $2.048(1.225-4.091)$ & 0.014 \\
\hline
\end{tabular}

$\mathrm{Cl}$ - confidence interval; $\mathrm{CK}-\mathrm{MB}$ - creatine kinase myocardial band; HDL — how density lipoprotein cholesterol; HR - hazard ratio; $\mathrm{MHR}$ - monocyte to high density lipoprotein cholesterol ratio; WBC — white blood cell 
Table 4. Significant predictors of in-hospital major adverse cardiac events in univariable and multivariable Cox regression analyses.

\begin{tabular}{|c|c|c|c|c|}
\hline \multirow[t]{2}{*}{ Variables } & \multicolumn{2}{|c|}{ Univariable } & \multicolumn{2}{|c|}{ Multivariable } \\
\hline & HR (95\% Cl) & $\mathbf{P}$ & HR (95\% CI) & $\mathbf{P}$ \\
\hline Age & $0.985(0.954-1.017)$ & 0.349 & - & - \\
\hline Male gender & $0.897(0.349-2.307)$ & 0.822 & - & - \\
\hline Diabetes mellitus & $1.645(0.747-3.624)$ & 0.217 & - & - \\
\hline Hypertension & $0.720(0.489-1.160)$ & 0.108 & - & - \\
\hline Smoking & $1.070(0.549-2.086)$ & 0.842 & - & - \\
\hline Glucose & $0.998(0.993-1.002)$ & 0.289 & - & - \\
\hline Ejection fraction & $1.004(0.977-1.032)$ & 0.775 & - & - \\
\hline Cholesterol & $1.007(0.998-1.016)$ & 0.130 & - & - \\
\hline HDL & $0.961(0.918-1.006)$ & 0.092 & $0.976(0.942-1.010)$ & 0.162 \\
\hline Triglyceride & $0.997(0.993-1.001)$ & 0.153 & - & - \\
\hline WBC & $1.092(1.001-1.191)$ & 0.046 & $1.073(1.000-1.152)$ & 0.049 \\
\hline Monocyte & $1.584(0.962-1.632)$ & 0.073 & $1.488(0.882-1.742)$ & 0.102 \\
\hline Hemoglobin & $0.921(0.746-1.139)$ & 0.449 & - & - \\
\hline Platelet & $1.001(0.997-1.005)$ & 0.611 & - & - \\
\hline Peak CK-MB & $0.999(0.998-1.001)$ & 0.456 & - & - \\
\hline MHR & 1.476 (1.116-1.944) & 0.030 & 1.501 (1.015-1.993) & 0.022 \\
\hline
\end{tabular}

$\mathrm{Cl}$ - confidence interval; CK-MB — creatine kinase myocardial band; HDL - how density lipoprotein cholesterol; HR - hazard ratio; $\mathrm{MHR}$ - monocyte to high density lipoprotein cholesterol ratio; WBC — white blood cell

Table 5. Significant predictors of major adverse cardiac events at 5-year follow-up in univariable and multivariable Cox regression analyses.

\begin{tabular}{|c|c|c|c|c|}
\hline \multirow[t]{2}{*}{ Variables } & \multicolumn{2}{|c|}{ Univariable } & \multicolumn{2}{|c|}{ Multivariable } \\
\hline & HR (95\% Cl) & $\mathbf{P}$ & HR (95\% Cl) & $\mathbf{P}$ \\
\hline Age & $1.014(0.997-1.030)$ & 0.102 & - & - \\
\hline Male gender & $0.860(0.558-1.326)$ & 0.494 & - & - \\
\hline Diabetes mellitus & $1.138(0.762-1.698)$ & 0.528 & - & - \\
\hline Hypertension & $1.084(0.883-1.330)$ & 0.440 & - & - \\
\hline Smoking & $1.413(1.09-1.979)$ & 0.044 & $1.294(0.945-1.773)$ & 0.108 \\
\hline Glucose & $1.003(1.001-1.005)$ & 0.010 & $1.004(0.996-1.012)$ & 0.080 \\
\hline Ejection fraction & $0.981(0.967-0.995)$ & 0.007 & $0.980(0.967-0.993)$ & 0.003 \\
\hline Cholesterol & $1.001(0.997-1.006)$ & 0.538 & - & - \\
\hline $\mathrm{HDL}$ & $1.000(0.980-1.0120)$ & 1.000 & - & - \\
\hline Triglyceride & $1.000(0.998-1.002)$ & 0.916 & - & - \\
\hline WBC & $1.020(0.974-1.069)$ & 0.400 & - & - \\
\hline Monocyte & $1.427(0.973-1.988)$ & 0.102 & - & - \\
\hline Hemoglobin & $0.916(0.842-1.011)$ & 0.082 & $0.909(0.829-0.996)$ & 0.041 \\
\hline Platelet & $1.001(0.999-1.003)$ & 0.242 & - & - \\
\hline Peak CK-MB & $1.000(0.999-1.001)$ & 0.487 & - & - \\
\hline MHR & $1.248(1.020-1.528)$ & 0.031 & $1.285(1.064-1.552)$ & 0.009 \\
\hline
\end{tabular}

$\mathrm{Cl}$ - confidence interval; CK-MB — creatine kinase myocardial band; HDL - how density lipoprotein cholesterol; HR - hazard ratio; $\mathrm{MHR}$ - monocyte to high density lipoprotein cholesterol ratio; WBC - white blood cell 


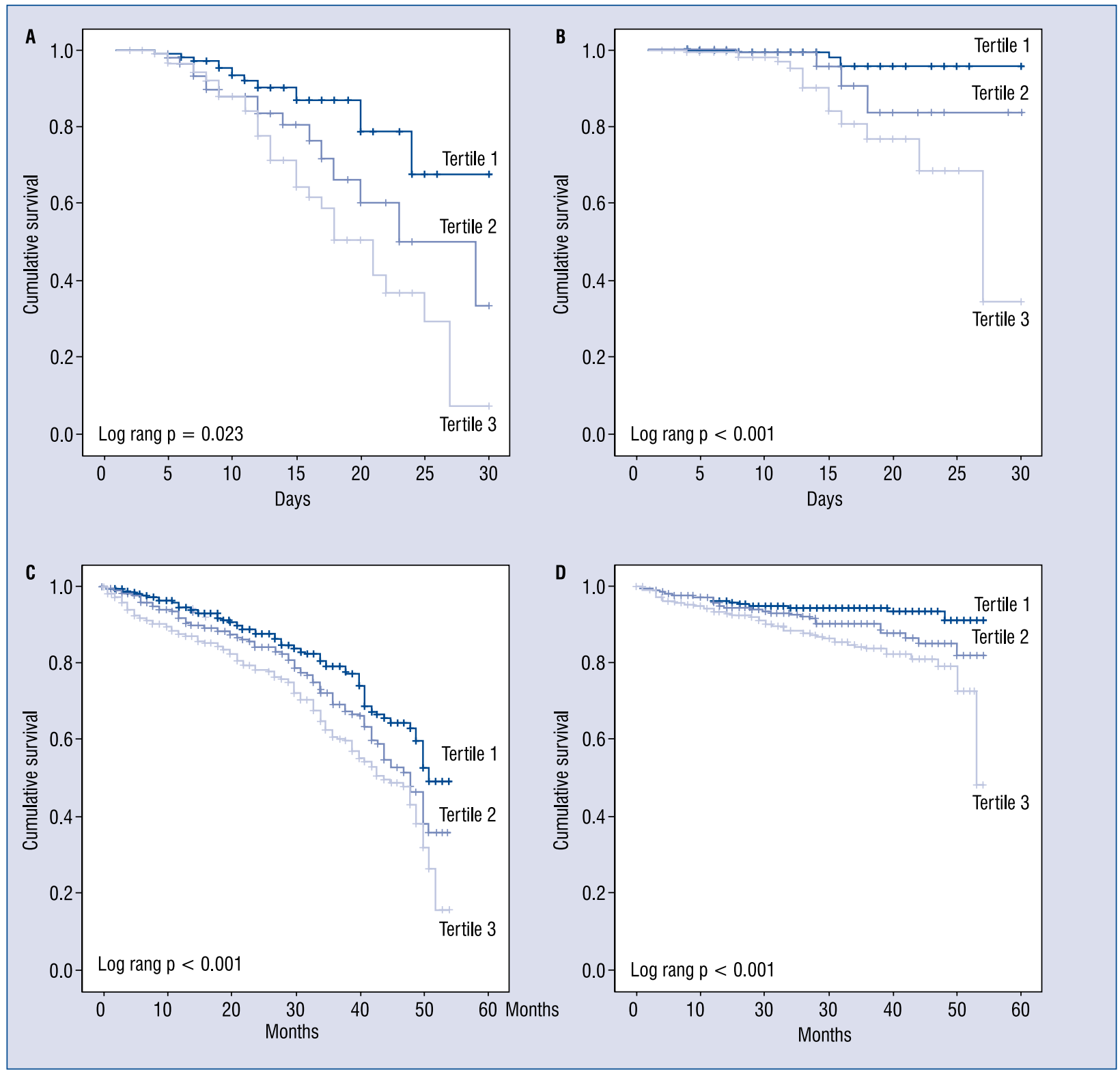

Figure 1. Kaplan-Meier cumulative survival curves for in-hospital and 5-year mortality and major adverse cardiac events (MACE), according to monocyte to high density lipoprotein cholesterol tertiles; A. In-hospital MACE; B. In-hospital mortality; C. 5-year MACE; D. 5-year mortality.

a valuable prognosis marker in cardiology and deserve to be investigated in other cardiovascular conditions.

\section{Limitations of the study}

The present study has some limitations. First, it is a retrospective and single center study. In addition, a single MHR value was used for analysis rather than a temporal trend. Another limitation of the study is that other inflammation and oxidative stress markers, such as C-reactive protein, were not used. In addition, the use of newer drugs such as prasugrel and ticagrelor instead of clopidogrel may alter the results of the study.

\section{Conclusions}

In conclusion, results of the present study showed that MHR is an independent predictor of in-hospital and 5-year MACE and mortality in STEMI patients. Prospective studies are needed to confirm our findings and elucidate the value of MHR in prediction of prognosis after STEMI. 


\section{Conflict of interest: None declared}

\section{References}

1. Libby P. Inflammation in atherosclerosis. Nature, 2002; 420: 868-874.

2. Heitzer T, Schlinzig T, Krohn K et al. Endothelial dysfunction, oxidative stress, and risk of cardiovascular events in patients with coronary artery disease. Circulation, 2001; 104: 2673-2678.

3. Ancuta P, Wang J, Gabuzda D. CD16+ monocytes produce IL-6, CCL2, and matrix metalloproteinase-9 upon interaction with CX3CL1-expressing endothelial cells. J Leukoc Biol, 2006; 80: 1156-1164.

4. Karabacak M, Kahraman F, Sert M et al. Increased plasma monocyte chemoattractant protein-1 levels in patients with isolated low highdensity lipoprotein cholesterol. Scand J Clin Lab Invest, 2015; 75: 327-332.

5. Zhang Y, Zanotti I, Reilly MP et al. Overexpression of apolipoprotein A-I promotes reverse transport of cholesterol from macrophages to feces in vivo. Circulation, 2003; 108: 661-663.

6. Kanbay M, Solak Y, Unal HU et al. Monocyte count/HDL cholesterol ratio and cardiovascular events in patients with chronic kidney disease. Int Urol Nephrol, 2014; 46: 1619-1625. doi: 10.1007/s11255-014-0730-1.

7. Canpolat U, Çetin EH, Cetin S et al. Association of Monocyteto-HDL Cholesterol Ratio with Slow Coronary Flow is Linked to Systemic Inflammation. Clin Appl Thromb Hemost, 2015; Jul 1. pii: 1076029615594002. [Epub ahead of print].

8. Kundi H, Gok M, Kiziltunc $\mathrm{E}$ et al. Relation between monocyte to high-density lipoprotein cholesterol ratio with presence and severity of isolated coronary artery ectasia. Am J Cardiol, 2015; 116: 1685-1659.

9. Canpolat U, Aytemir K, Yorgun $\mathrm{H}$ et al. The role of preprocedural monocyte-to-high-density lipoprotein ratio in prediction of atrial fibrillation recurrence after cryoballoon-based catheter ablation. Europace, 2015; 17: 1807-1815.

10. Cetin EH, Cetin MS, Canpolat U et al. Monocyte/HDL-cholesterol ratio predicts the definite stent thrombosis after primary percutaneous coronary intervention for ST-segment elevation myocardial infarction. Biomark Med, 2015; 9: 967-977.

11. Karataş MB, Çanga Y, Özcan KS et al. Monocyte to high-density lipoprotein ratio as a new prognostic marker in patients with STsegment elevation myocardial infarction undergoing primary percutaneous coronary intervention. Am J Emerg Med, 2015; Oct 27. pii: S0735-6757(15)00939-0. doi: 10.1016/j.ajem.2015.10.049. [Epub ahead of print].

12. Steinberg D, Witztum JL. Is the oxidativemodification hypothesis relevant to human atherosclerosis? Do the antioxidant trials conducted to date refute the hypothesis? Circulation, 2002; 105: 2107-2111.

13. Moreno PR, Purushothaman KR, Fuster V et al. Intimomedial interface damage and adventitial inflammation is increased beneath disrupted atherosclerosis in the aorta: implications for plaque vulnerability. Circulation, 2002; 105: 2504-2511.

14. Kaikita $\mathrm{K}$, Ogawa $\mathrm{H}$, Yasue $\mathrm{H}$ et al. Tissue factor expression on macrophages in coronary plaques in patients with unstable angina. Arterioscler Thromb Vasc Biol, 1997; 17: 2232-2237.

15. Johnsen SH, Fosse E, Joakimsen O et al. Monocyte count is a predictor of novel plaque formation: A 7-year follow-up study of 2610 persons without carotid plaque at baseline the Tromso Study. Stroke, 2005; 36: 715-719.

16. Gratchev A, Sobenin I, Orekhov A et al. Monocytes as a diagnostic marker of cardiovascular diseases. Immunobiology, 2012; 217: 476-482.

17. Cockerill GW, Rye KA, Gamble JR et al. High-density lipoproteins inhibit cytokine-induced expression of endothelial, cell adhesion molecules. Arterioscler Thromb Vasc Biol, 1995; 15: 1987-1994.

18. Murphy AJ, Chin-Dusting JP, Sviridov D et al. The anti inflammatory effects of high Density lipoproteins. Curr Med Chem, 2009; 16: 667-675.

19. Murphy AJ, Woollard KJ. High-density lipoprotein: a potent inhibitor of inflammation. Clin Exp Pharmacol Physiol, 2010; 37: 710-718.

20. Yvan-Charvet L, Pagler T, Gautier EL et al. ATP-binding cassette transporters and HDL suppress Hematopoietic stem cell proliferation. Science, 2010; 328: 1689-1693.

21. Khovidhunkit W, Memon RA, Feingold KR et al. Infection and inflammation-induced proatherogenic changes of lipoproteins. J Infect Dis, 2000; 181: S462-S472.

22. Cabana VG, Lukens JR, Rice KS et al. HDL content and composition in acute phase response in three species: triglyceride enrichment of HDL a factor in its decrease. J Lipid Res, 1996; 37: 2662-2674.

23. Van Lenten BJ, Wagner AC, Nayak DP et al. HDL loses its anti-inflammatory properties during acute influenza $\mathrm{A}$ infection. Circulation, 2001; 103: 2283-2288.

24. Artl A, Marsche G, Lestavel S et al. Role of serum amyloid A during metabolism of acute-phase HDL by macrophages. Arterioscler Thromb Vase Biol, 2000; 20: 763-772. 